\title{
REFLEXÕES DE ESPECIALISTAS SOBRE ATIVIDADES DE PROJETO CONCEITUAL NO DESENVOLVIMENTO DE PSS
}

Caio Augusto Nunes Marques (caio.nunes.marques@gmail.com) - Departamento de Engenharia de Produção, Escola de Engenharia, Universidade de São Paulo

Henrique Rozenfeld (roz@usp.br) - Departamento de Engenharia de Produção, Escola de Engenharia, Universidade de São Paulo

\section{RESUMO}

Ao longo das duas últimas décadas, representantes da comunidade acadêmica e da indústria têm percebido que produzir e vender bens físicos já não garante a competitividade das empresas. Uma alternativa que surge é o oferecimento de uma combinação de produtos e serviços em uma oferta que entrega valor provendo uma função ou resultado. Essa oferta de valor é conhecida como Sistema Produto-Serviço (PSS - Product-Service System) e seu processo de desenvolvimento tem sido objeto de estudo de diversos autores. Existem modelos de processo genéricos de desenvolvimento de PSS propostos na literatura, que servem como referência para que uma empresa defina seu modelo específico. No entanto, cada um desses modelos genéricos possui particularidades e vieses, logo nenhum deles pode ser usado como referência para toda e qualquer empresa defina seu modelo específico. Existe uma pesquisa de doutorado em andamento com o objetivo de propor um framework para criar modelos de processo específicos de desenvolvimento de PSS. Nesta pesquisa foram avaliados quinze modelos genéricos, a partir dos quais foram identificadas 329 atividades que podem ser realizadas para se desenvolver PSS (atividades-síntese). O trabalho descrito neste artigo está inserido no contexto da pesquisa de doutorado, e tem como objetivo testar a versão inicial do framework. Para tanto foram realizados oito focus groups com a participação de vinte e sete representantes de treze empresas e três acadêmicos de duas universidades. Os participantes tinham que selecionar quais das vinte e quatro atividades-sintese executariam na fase de "projeto conceitual" do desenvolvimento de PSS apresentado em um caso. Os participantes podiam incluir novas atividades ao conjunto inicial, o que levou à proposição de mais vinte e seis atividades. A versão inicial do framework mostrou-se de fácil utilização para os participantes e fomentou debates e reflexões acerca do desenvolvimento de PSS. Foi possível notar que os participantes ainda não têm um consenso com relação à relevância da sustentabilidade no desenvolvimento de PSS, apesar de ser um assunto largamente discutido na academia. As principais limitações decorrem da aplicação ter sido realizada em um ambiente simulado e da pouca experiência dos participantes no desenvolvimento de PSS.

Palavras-chave: Servitização; sistemas produto-serviço; modelos de processo

Área: Desenvolvimento de Sistemas Produto-Serviço (PSS)

\section{INTRODUÇÃO}

Tem ganhado espaço tanto na academia quanto na indústria o entendimento de que a simples produção de bens físicos, com posterior transferência de propriedade via comercialização já não mais atende plenamente aos anseios dos clientes. O que eles buscam é a solução de um problema (BOEHM; THOMAS, 2013, p. 245). Uma alternativa para as empresas é migrar do business-as-usual, adotar uma estratégia de servitização e, consequentemente, disponibilizar 
uma combinação de produtos e serviços via um Sistema Produto-Serviço (PSS - ProductService System) no mercado (MANZINI; VEZZOLI, 2002, p. 16).

Com um PSS, a empresa passa a oferecer valor não somente via transferência de propriedade de um bem físico, mas combina produtos e serviços para prover ao cliente uma função ou resultado requerido (ALONSO-RASGADO; THOMPSON; ELFSTRÖM, 2004, p. 515; BOEHM; THOMAS, 2013, p. 245; MANZINI; VEZZOLI, 2002, p. 3; QU et al., 2016, p. 1). As necessidades dos clientes são satisfeitas pela função recebida e não pela posse de produtos (BOEHM; THOMAS, 2013, p. 245; MANZINI; VEZZOLI, 2003, p. 851; MONT, 2002, p. 238; TUKKER, 2015, p. 76).

Em muitos casos, a posse dos produtos que compõem o PSS permanece com o provedor, o que implica em adotar estratégias de fim de vida como reuso e remanufatura (BARQUET et al., 2013, p. 694) que, por sua vez, contribuem para a desmaterialização da economia (MONT, 2002, p. 237). Com relação à competitividade, as empresas passam a diferenciar sua oferta de valor dos concorrentes (AURICH; FUCHS; WAGENKNECHT, 2006, p. 1480), oferecendo soluções customizadas (TUKKER; TISCHNER, 2006b, p. 1553) e beneficiando-se das margens de lucro normalmente superiores em virtude do oferecimento de serviços (MEIER; ROY; SELIGER, 2010, p. 608; PERUZZINI; MARILUNGO; GERMANI, 2014, p. 956). Em suma, existem diversos benefícios esperados decorrentes do oferecimento de PSS para o provedor, clientes, meio ambiente e governo (ALONSO-RASGADO; THOMPSON, 2006, p. 510; ALONSO-RASGADO; THOMPSON; ELFSTRÖM, 2004, p. 516; BAINES et al., 2007, p. 1548; MONT, 2002, p. 239; UNEP, 2001, p. 4).

O uso de um modelo de processo é considerado uma boa prática para guiar o desenvolvimento de PSS (AURICH; FUCHS; WAGENKNECHT, 2006, p. 1483; VAN HALEN; MANZINI; WIMMER, 2005). Para tanto, existem diversos modelos de processo de desenvolvimento de PSS propostos na literatura, conforme indicam as revisões feitas por Tukker e Tischner (2006a, p. 100), Clayton, Backhouse e Dani (2012, p. 275) e Vasantha et al. (2012, p. 636). Os modelos revisados nestes trabalhos são genéricos, isto é, servem como uma referência para definir os modelos específicos que as empresas empregam para desenvolver PSS. Para os modelos específicos, dentre outros usos, destaca-se o apoio ao planejamento de projetos de desenvolvimento (COSTA, 2016, p. 22).

Os modelos genéricos propostos na literatura possuem diferentes focos e particularidades (MARQUES et al., 2016, p. 146). Por exemplo, Alonso-Rasgado e Thompson (2006) propõem um modelo voltado para o desenvolvimento de PSS sob encomenda; Brezet et al. (2001), Luiten, Knot e Horst (2001) e van Halen, Manzini e Wimmer (2005) apresentam modelos que enfatizam a perspectiva ambiental do desenvolvimento de PSS; Sakao e Shimomura (2007) preocupam-se mais com o desenvolvimento de serviços; e o modelo de Nguyen et al. (2014) incorpora a visão de várias disciplinas, mas com um viés maior em aspectos técnicos do desenvolvimento. Assim, não há um modelo que seja genérico o suficiente para servir como referência a toda e qualquer empresa definir seu modelo de processo específico de desenvolvimento de PSS.

Para cobrir esta lacuna, existe uma pesquisa de doutorado em andamento com o objetivo de propor um framework para criar modelos de processo específicos de desenvolvimento de PSS. Nesta pesquisa foram avaliados quinze modelos de processo genéricos de desenvolvimento de PSS propostos na literatura. Tais modelos foram analisados individualmente e comparados de forma que foram identificadas 329 possíveis atividades de desenvolvimento de PSS e suas respectivas descrições (doravante chamadas de atividades-síntese). A proposta é que essas atividades sirvam como ponto de partida para que uma empresa defina seu modelo específico, ao invés de ficar limitada à escolha de um modelo genérico, que pode não atender às suas 
características. Posteriormente serão associados métodos e ferramentas que podem ser utilizados para executar as atividades-síntese. $\mathrm{O}$ trabalho aqui descrito faz parte da pesquisa de doutorado e tem como objetivo testar a versão inicial do framework, considerando somente as atividades-síntese. A proposta do framework é de não utilizar o conceito de fases, mas de áreas de conhecimento. Contudo, optou-se por selecionar 24 atividades-síntese restritas à fase "Projeto Conceitual", para viabilizar o teste devido às restrições de tempo.

Na próxima seção apresenta-se o método de pesquisa utilizado para testar o framework. Em seguida são apresentados os resultados do trabalho, com ênfase nas atividades-síntese selecionadas, nas novas atividades sugeridas e nas reflexões observadas. Por fim são apresentados os principais achados, destacadas as limitações e apontados os passos futuros da pesquisa de doutorado.

\section{MÉTODO DE PESQUISA}

O método de pesquisa escolhido para realizar o teste do framework foi o focus group. Um focus group ou grupo focal ou grupo de foco, é um método de investigação e coleta de dados que pode ser empregado em diferentes fases da pesquisa (SILVA; VELOSO; KEATING, 2014, p. 177). O focus group foi estruturado de acordo com a proposta apresentada por Morgan (1998, p. 65). O autor sugere que o focus group seja organizado em quatro etapas sequenciais (vide Figura 1), que serão descritas nas subseções a seguir.

Figura 1 - Etapas de um focus group.

Planejar focus
group $\longrightarrow \begin{gathered}\text { Recrutar } \\ \text { participantes }\end{gathered} \longrightarrow \begin{gathered}\text { Moderar focus } \\ \text { group }\end{gathered} \longrightarrow \begin{gathered}\text { Analisar dados } \\ \text { e divulgar } \\ \text { resultados }\end{gathered}$

Fonte: Morgan (1998, p. 65).

\subsection{Planejar focus group}

Trabalhar com todas as atividades-síntese seria inviável em virtude de seu número elevado (329). Decidiu-se então restringir o teste à fase de "projeto conceitual" e utilizar uma amostra de vinte e quatro atividades que poderiam ser realizadas nesta fase. A fase de "projeto conceitual" foi delimitada considerando que algumas entregas (ideias de soluções; portfólio de produtos da empresa; estratégia da empresa; clientes-alvo; análise de competidores; análise de soluções similares; modelo de negócios atual; parceiros atuais; necessidades dos clientes) já haviam sido finalizadas. O resultado e entrega final da fase seria a arquitetura do PSS.

O focus group foi organizado de forma a contar com três papéis principais. O moderador, que era responsável por instruir, mediar as discussões e tirar dúvidas. O monitor, responsável por observar as discussões e anotar os pontos interessantes que conseguiu observar no grupo ao qual estava dedicado. O participante, com o papel de interagir com os demais membros do grupo e debater sobre os conteúdos disponíveis, notadamente as atividades-síntese.

O objetivo definido para os participantes durante o focus group foi preparar um plano para a fase de "projeto conceitual" de um PSS em desenvolvimento. A execução do focus group foi definida para ocorrer em quatro passos: Passo 01: Avaliar material preparatório; Passo 02: Selecionar atividades; Passo 03: Adicionar atividades e Passo 04: Excluir atividades.

Ainda na etapa "Planejar focus group", foi criado o material de apoio para o focus group. O principal material criado foram as cartas que continham o conteúdo das atividades-síntese (nome e descrição) e tinham como função estimular o debate entre os participantes. A Figura 2 representa uma dessas cartas. Na parte frontal apresentam-se o nome da atividade ao centro, 
um código da atividade no canto superior direito (para facilitar a identificação) e uma quantidade de estrelas que varia entre uma e três, correspondente ao custo da atividade. $\mathrm{Na}$ parte frontal inferior era afixada uma etiqueta para que os participantes pudessem votar se concordam ou não com a seleção daquela atividade-síntese. No verso repete-se o nome da atividade e sua descrição. Adicionalmente ainda foram criados os seguintes materiais de apoio: resumo do caso de desenvolvimento de um PSS fictício; arquitetura do PSS do caso; pôster da fase de "projeto conceitual" e roteiro de observação.

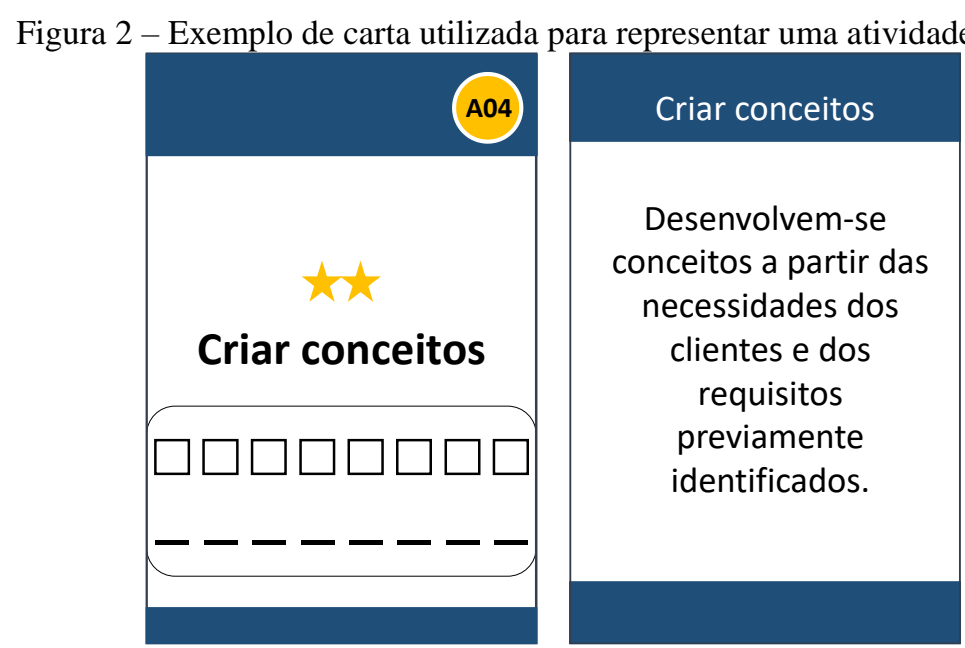

Antes de realizar o focus group com profissionais da indústria, foram realizadas duas simulações internas sequenciais (pré-teste e teste) com a participação de professores e pósgraduandos de um grupo de pesquisa focado em desenvolvimento de produtos. No "pré-teste" os três participantes (pós-graduandos) passaram pelos quatro passos descritos anteriormente e fizeram sugestões com relação às atividades escolhidas, suas descrições e o tempo reservado para cada passo. As sugestões foram levadas em consideração e aplicadas no "teste", que contou com a participação de sete pesquisadores (dois professores e cinco pós-graduandos). Do mesmo modo, as sugestões feitas durante o "teste" foram consideradas na execução dos focus group. Após o "pré-teste" e o "teste", foram decididas quais seriam as vinte e quatro atividades-síntese a serem consideradas pelos profissionais da indústria (Quadro 1).

Quadro 1 - Atividades selecionadas para serem avaliadas no focus group com os participantes das empresas.

\begin{tabular}{c} 
Atividades \\
\hline A01 - Estabelecer requisitos; A02 - Determinar requisitos críticos; A03 - Desenvolver funções essenciais; A04 \\
- Criar conceitos; A05 - Selecionar conceitos; A06 - Testar conceitos; A07 - Definir serviços; A08 - Pesquisar \\
tecnologias disponíveis; A09 - Definir ciclo de vida do PSS; A10 - Criar arquitetura dos serviços; A11 - Criar \\
arquitetura dos produtos; A12 - Consolidar arquitetura do PSS; A13 - Realizar análise de riscos; A14 - Avaliar \\
viabilidade técnica; A15 - Criar modelos de negócios; A16 - Avaliar sustentabilidade; A17 - Avaliar viabilidade \\
econômico-financeira; A18 - Estabelecer parcerias; A19 - Comunicar decisões de projeto; A20 - Apresentar \\
arquitetura aos stakeholders; A21 - Analisar custos; A22 - Avaliar uso de DfX; A23 - Definir estratégias de fim \\
de vida; A24 - Registrar lições aprendidas \\
\hline
\end{tabular}

\subsection{Recrutar participantes}

Os participantes indicados para o focus group seriam os profissionais que definem os modelos específicos, gerenciam o processo de desenvolvimento ou mesmo que atuem e possuam uma visão abrangente do processo. Assim, foram convidados profissionais que trabalham com desenvolvimento de produtos, já desenvolveram ou pretendem desenvolver PSS. Foi possível recrutar 27 profissionais que trabalham em treze empresas, aos quais se juntaram mais três acadêmicos de duas universidades. A Tabela 1 apresenta o setor de atuação de cada uma das treze empresas que enviaram seus profissionais para participar do focus group. 
Tabela 1 - Perfil das empresas que participaram do focus group.

\begin{tabular}{cccc}
\hline Empresa & Setor & Empresa & Setor \\
\hline A & Sistemas e partes automobilísticas & H & Energia e açúcar \\
B & Equipamentos médicos e de saúde & I & Máquinas de transformação de plástico \\
$\mathrm{C}$ & Sistemas e partes automobilísticas & J & Cosméticos \\
D & Máquinas e soluções agropecuárias & $\mathrm{K}$ & Máquinas de corte \\
$\mathrm{E}$ & Compressores & $\mathrm{L}$ & Sistemas e partes automobilísticas \\
$\mathrm{F}$ & Aeronáutica & $\mathrm{M}$ & Embalagens biodegradáveis \\
$\mathrm{G}$ & Equipamentos médicos e de saúde & & \\
\hline
\end{tabular}

\subsection{Moderar focus group}

Os trinta participantes foram organizados em oito focus groups simultâneos, com duração de uma hora e trinta minutos. Os grupos foram formados com o objetivo de manter homogeneidade entre os participantes. Para tanto levou-se em consideração o nível hierárquico dos participantes em suas respectivas empresas, de modo a evitar que houvesse predominância de um participante sobre os demais em função de sua posição na empresa.

Cada grupo foi acompanhado por um monitor previamente treinado. Paralelamente, três moderadores deslocavam-se entre os grupos, debatendo questões que os monitores não podiam solucionar.

Os grupos receberam vinte e quatro cartas com as atividades-síntese apresentadas no Quadro 1 , bem como os materiais adicionais descritos na seção "Planejar focus group". Durante o focus group, os participantes debatiam se as atividades-síntese do framework eram pertinentes e suficientes para executar o "Projeto conceitual" do PSS do caso, selecionando algumas delas para compor um plano de projeto de desenvolvimento do PSS fictício apresentado no "resumo do caso", restrito à fase "Projeto Conceitual" (como ilustrado na Figura 3). Eles debatiam cada atividade, manifestavam a opinião individualmente e selecionavam aquelas que recebiam o voto de ao menos metade dos participantes de cada grupo.

\subsection{Analisar dados e divulgar resultados}

Esta etapa foi feita, de acordo com a classificação de Morgan (1998, p. 70), por meio de anotações das discussões. As anotações eram registradas pelos monitores em cada grupo. Depois dos focus group, foram feitas reuniões individuais com cada monitor para sanar dúvidas relativas às anotações e identificar novas observações que os monitores não registraram no roteiro de observação, mas que perceberam durante os focus groups. Em seguida os dados foram tabulados e os resultados são apresentados na próxima seção.

Figura 3 - Seleção de atividades em um dos focus groups.

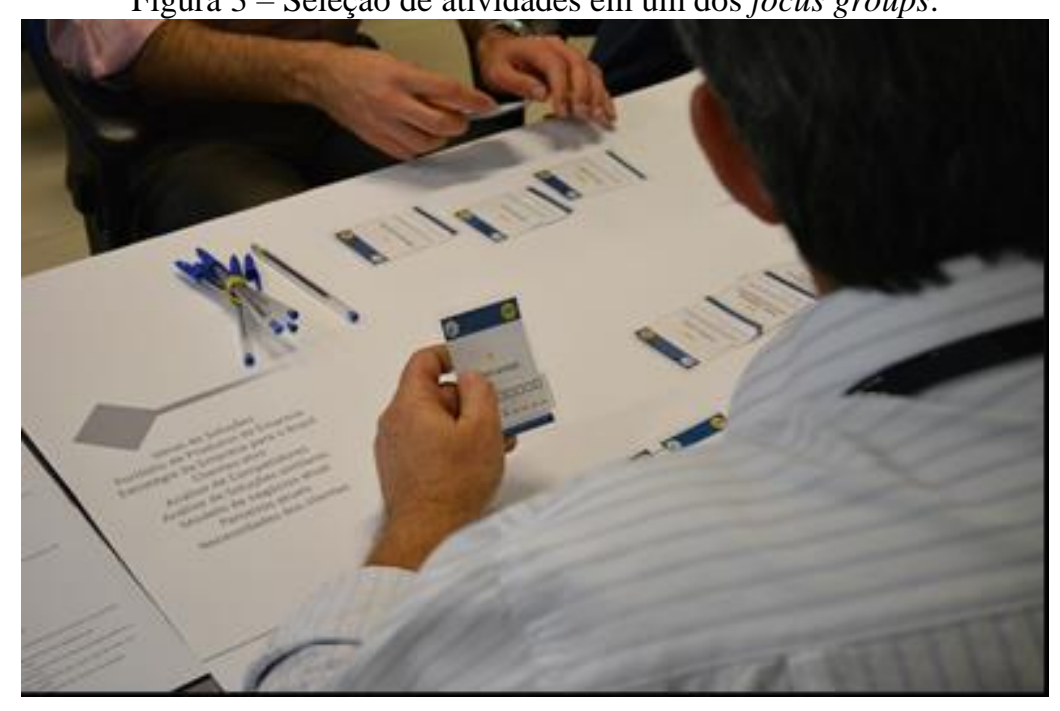




\section{RESULTADOS}

\subsection{Passo 02: Selecionar atividades}

Como não havia limite de atividades que poderiam ser selecionadas, todas foram escolhidas pela maioria dos grupos. A Figura 4 ilustra o percentual de grupos que selecionou cada uma das atividades. As atividades são representadas pelos mesmos códigos descritos no Quadro 1. Das vinte e quatro atividades-síntese disponíveis, dez delas (A02, A03, A04, A07, A12, A13, A16, A19, A20 e A24) foram selecionadas por todos os grupos e outras dez (A01, A05, A06, A09, A10, A14, A17, A18, A21 e A23) foram selecionadas por sete dos oito grupos. Isso significa que ampla maioria das atividades-síntese apresentadas foram consideradas necessárias para a realizar o "Projeto conceitual" do PSS do caso.

Figura 4 - Percentual de focus groups que selecionaram ou não selecionaram cada uma das 24 atividades-síntese

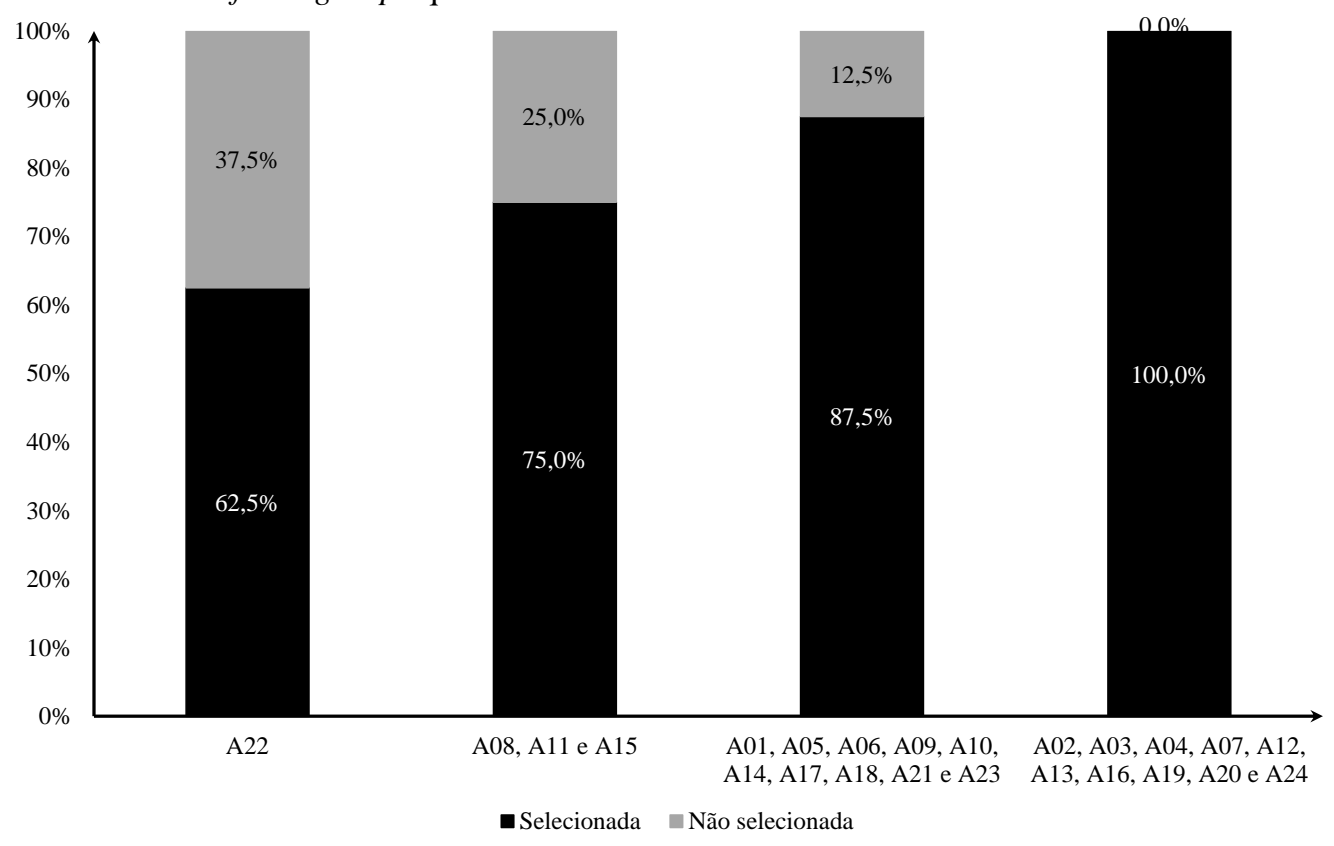

As atividades A08 - Pesquisar tecnologias disponíveis, A11 - Criar arquitetura dos produtos e A15 - Criar modelos de negócios não foram selecionadas por dois grupos. No caso das duas primeiras atividades, foi possível observar três abordagens distintas nos grupos que rejeitaram ao menos uma dessas atividades. O grupo G03 considerou que, como apresentado no "resumo do caso", a empresa vendia produtos e passaria a oferecer um PSS, os produtos já eram existentes, logo a arquitetura dos produtos também seria e as tecnologias já estariam embutidas nos produtos. O grupo G04 também entendeu a arquitetura dos produtos como o grupo G03, mas avaliou que seria necessário pesquisar novas tecnologias. Por fim, o grupo G08 não viu necessidade em pesquisar tecnologias disponíveis, mas definiu que seria necessário criar a arquitetura dos produtos. Essas conclusões indicam uma mentalidade centrada ainda no desenvolvimento de produtos.

A atividade A22 - Avaliar uso de DfX foi a menos selecionada pelos grupos, com três dos oito grupos não a selecionando (G03, G04 e G07). Havia participantes em grupos (G02, G03 e G05) que afirmaram desconhecer o termo Design for $X$, o que pode indicar um distanciamento entre universidade e empresas, por ser um termo largamente difundido na academia.

\subsection{Passo 03: Adicionar atividades}

Após selecionarem as atividades-síntese, os participantes tiveram um tempo para adicionar 
novas atividades que consideravam importantes na fase "projeto conceitual" no desenvolvimento de PSS que não estavam previstas dentre as vinte e quatro apresentadas no Quadro 1. Os participantes consideraram experiências pregressas no desenvolvimento de produtos ou de PSS para sugerir as novas atividades apresentadas no Quadro 2. As atividades são identificadas com um código. Os três primeiros dígitos identificam o grupo e o último dígito, representa a sequência com que foram citadas.

Quadro 2 - Atividades adicionadas no focus group pelos participantes das empresas.

\begin{tabular}{c} 
Atividades \\
\hline G011 - Treinamento; G012 - Definir indicadores de desempenho-chave; G013 - Compliance; G021 - Incorporar \\
feedback de mercado; G022 - Identificar potenciais patrocinadores; G023 - Definir requisitos de usuários \\
finais/clientes; G031 - Survey satisfação; G032 - Analisar mão-de-obra; G041 - Definição da infraestrutura \\
necessária para o PSS; G042 - Envolver o cliente na definição do modelo de negócios; G043 - Testar o modelo \\
de negócios; G044 - Definir ecossistema de negócios (regionalização)/parceria operacional; G045 - Avaliar \\
precificação/tarifas fiscais; G046 - Definição regras contábeis; G051 - Analisar disponibilidade de recursos \\
humanos capacitados; G052 - Levantar requisitos legais; G061 - User experience feedback; G062 - Plano de \\
comunicação; G063 - Análise de recursos (corrente crítica para otimização); G064 - Análise dos stakeholders; \\
G071 - Análise de concorrência; G072 - Análise de mercado; G081 - Plano macro de processo
\end{tabular}

Ao avaliar as atividades apresentadas no Quadro 2, nota-se a grande importância atribuída ao entendimento do cliente e do mercado (atividades G021, G023, G031, G042, G043, G061, G062, G071 e G072), pessoas (G011, G032 e G051), requisitos contábeis/financeiros/legais (G045, G046 e G052) e gestão do processo (G012, G013 e G081). Destaca-se ainda a sugestão da atividade G044 - Definir ecossistema de negócios (regionalização)/parceria operacional, que é pertinente em um país de grandes dimensões territoriais como o Brasil.

Aqui é importante destacar que não significa que as atividades propostas pelos participantes não constam entre as 329 identificadas na literatura. Na verdade, eles só tiveram acesso à amostra de 24 atividades pertinentes à fase "projeto conceitual". Assim as contribuições foram feitas com base nessas 24 atividades e não tendo o conhecimento do conjunto total. Logo, existem atividades propostas pelos participantes que estão em outras fases que não o "projeto conceitual" (Ex.: G011, G023 e G062) e algumas que não constam entre as 329 atividades identificadas na literatura (Ex.: G046).

\subsection{Reflexões}

Destacam-se as discussões com relação à sustentabilidade, um tema fundamental na teoria de PSS, e representado no focus group por duas atividades A16 - Avaliar sustentabilidade e A23 - Definir estratégias de fim de vida. Apesar de terem sido selecionadas por, respectivamente, $100 \%$ e $87,5 \%$ dos grupos (Figura 4), os grupos apresentaram opiniões conflitantes com relação à importância da Sustentabilidade. O grupo G01 entende que devem ser considerados os três pilares da sustentabilidade (ambiental, social e econômico), como fariam ao desenvolver um PSS em suas empresas. O grupo G05 percebe uma importância relativa, sem considerá-la essencial. O grupo G03 percebe somente o pilar econômico relevante, considerando os demais não importantes no Brasil. Já os grupos G02 e G06 não consideram sustentabilidade importante, sendo que o G06 acredita que pensar em ciclo de vida e definir estratégias de fim de vida estão fora do escopo do desenvolvimento de PSS.

Um ponto fundamental percebido tanto no "pré-teste" quanto no "teste", mas que apenas o grupo G01 notou durante o focus group foi que as atividades-síntese apresentadas tinham foco majoritário em produtos e não em serviços. Isso faz sentido quando, ao analisar os modelos genéricos de desenvolvimento de PSS foram propostos, em grande número, por pesquisadores que trabalhavam previamente com desenvolvimento de produtos. 
Alguns debates transcendiam a fase de "projeto conceitual". O grupo G07 debateu a estrutura do processo de desenvolvimento de PSS. Na visão de seus membros o processo deve ser dividido em dois processos, que acontecem paralelamente: desenvolvimento de produto e desenvolvimento de serviço. Esta observação, no entanto, contradiz aquilo que é argumentado na literatura por autores como Cavalieri e Pezzotta (2012, p. 285) que entendem que o desenvolvimento de PSS deve ocorrem de forma integrada.

\section{CONCLUSÕES}

O trabalho aqui relatado é parte de uma pesquisa de doutorado em curso, cujo objetivo é propor um framework para criar modelos de processo específicos de desenvolvimento de PSS. O objetivo deste trabalho foi testar a versão inicial do framework. Para tanto foram realizados oito focus groups simultâneos com a participação de trinta profissionais, sendo vinte e sete de treze empresas e três de duas universidades.

Durante os focus groups os participantes tinham a missão de definir um plano de projeto para a fase de "projeto conceitual" de um projeto de desenvolvimento de um PSS fictício, apresentado em um caso. Para compor o plano eles debatiam e selecionavam entre vinte e quatro atividades-síntese provenientes de modelos de processo existentes na literatura de PSS. Eles ainda tinham a possibilidade de incluir novas atividades não previstas no conjunto inicial. Cada focus group era acompanhado por um monitor e existiam três moderadores que circulavam entre os grupos para facilitar as discussões.

Os participantes demonstraram facilidade no manuseio e discussão das atividades-síntese. Adicionalmente, o uso das atividades-síntese apresentadas no formato de cartas com nome e descrição da atividade (como na Figura 2) mostrou-se importante para orientar o trabalho e fomentar a discussão dentro de cada focus group. Isso significa que a seleção e debate sobre as atividades-síntese mostraram-se promissores para a definição dos modelos de processo específico de desenvolvimento de PSS.

Além de testar o uso do framework, foi possível identificar debates e reflexões entre os participantes dos focus groups que merecem destaque. Uma delas é que não há consenso entre eles com relação à importância da sustentabilidade no desenvolvimento de PSS. Enquanto na academia o debate está sobre o nível de importância, entre os participantes dos focus groups (majoritariamente de empresas) existiram opiniões em um espectro que vai desde a sustentabilidade ser fundamental até estar fora do escopo do desenvolvimento de PSS. Foi notado ainda que o conjunto de atividades-síntese (Quadro 1) apresentava forte viés de desenvolvimento de produto, versando pouco sobre desenvolvimento de serviço. O que se justifica quando se avalia que os autores dos modelos genéricos que serviram como base para a definição das atividades-síntese têm, em sua maioria, origem no desenvolvimento de produto. Por fim, um dos grupos discutiu ainda a possibilidade de que o desenvolvimento de PSS seja feito em dois processos paralelos (desenvolvimento de produto e desenvolvimento de serviço), o que já foi refutado pela literatura.

Uma limitação deste trabalho é que o framework foi testado em um ambiente simulado, pois os grupos eram formados por profissionais de diferentes empresas e o foco estava somente na fase de "projeto conceitual". Outra limitação é que, apesar de estarem habituados a desenvolver produtos, os participantes eram pouco experientes no desenvolvimento de PSS.

Uma alternativa para solucionar a primeira limitação é aplicar o framework no ambiente real de uma empresa, considerando todo o processo de desenvolvimento. Já a segunda limitação pode ser superada somente à medida que as empresas se habituem a desenvolver PSS, em especial com a parceria de pesquisadores. As próximas etapas da pesquisa de doutorado 
envolvem a identificação de métodos e ferramentas que podem ser utilizados para executar as atividades-síntese e o teste do framework completo.

\section{AGRADECIMENTOS}

À Coordenação de Aperfeiçoamento de Pessoal de Nível Superior (Capes), à Pró-Reitoria de Pesquisa da Universidade de São Paulo e ao processo no 2015/23094-6, Fundação de Amparo à Pesquisa do Estado de São Paulo (FAPESP).

\section{REFERÊNCIAS}

ALONSO-RASGADO, T.; THOMPSON, G. A rapid design process for Total Care Product creation. Journal of Engineering Design, v. 17, n. 6, p. 509-531, 2006.

ALONSO-RASGADO, T.; THOMPSON, G.; ELFSTRÖM, B.-O. The design of functional (total care) products. Journal of Engineering Design, v. 15, n. 6, p. 515-540, 2004.

AURICH, J. C.; FUCHS, C.; WAGENKNECHT, C. Life cycle oriented design of technical Product-Service Systems. Journal of Cleaner Production, v. 14, p. 1480-1494, 2006.

BAINES, T. S. et al. State-of-the-art in product-service systems. Proceedings of the Institution of Mechanical Engineers, Part B: Journal of Engineering Manufacture, v. 221, n. 10, p. 1543-1552, 1 jan. 2007.

BARQUET, A. P. B. et al. Employing the business model concept to support the adoption of product-service systems (PSS). Industrial Marketing Management, v. 42, n. 5, p. 693-704, jul. 2013.

BOEHM, M.; THOMAS, O. Looking beyond the rim of one's teacup: A multidisciplinary literature review of Product-Service Systems in Information Systems, Business Management, and Engineering \& Design. Journal of Cleaner Production, v. 51, p. 245-250, 2013.

BREZET, J. C. et al. The design of Eco-efficient Services. Delft: Delft University of Technology, 2001.

CAVALIERI, S.; PEZZOTTA, G. Product-Service Systems Engineering: State of the art and research challenges. Computers in Industry, v. 63, n. 4, p. 278-288, maio 2012.

CLAYTON, R. J.; BACKHOUSE, C. J.; DANI, S. Evaluating existing approaches to productservice system design: A comparison with industrial practice. Journal of Manufacturing Technology Management, v. 23, p. 272-298, 2012.

COSTA, D. G. The uses and users of design process models in organizations. [s.l.] Universidade de São Paulo, 2016.

LUITEN, H.; KNOT, M.; HORST, T. VAN DER. Sustainable Product-Service-Systems: the Kathalys method. Proceedings Second International Symposium on Environmentally Conscious Design and Inverse Manufacturing, p. 190-197, 2001.

MANZINI, E.; VEZZOLI, C. Product-Service Systems and Sustainability: Opportunities for sustainable solutions. Paris: United Nations Environment Programme, 2002.

MANZINI, E.; VEZZOLI, C. A strategic design approach to develop sustainable product service systems: examples taken from the "environmentally friendly innovation" Italian prize. Journal of Cleaner Production, v. 11, n. 8, p. 851-857, dez. 2003.

MARQUES, C. A. N. et al. Comparing PSS Design Models Based on Content Analysis. 
Procedia CIRP, v. 47, p. 144-149, 2016.

MEIER, H.; ROY, R.; SELIGER, G. Industrial Product-Service Systems-IPS2. CIRP Annals - Manufacturing Technology, v. 59, n. 2, p. 607-627, jan. 2010.

MONT, O. Clarifying the concept of product-service system. Journal of Cleaner Production, v. 10 , n. 3 , p. 237-245, jun. 2002.

MORGAN, D. L. The focus group guidebook. Thousand Oaks: Sage Publications, 1998.

NGUYEN, H. N. et al. Operationalizing IPS2 Development Process: A method for Realizing IPS2 Developments based on Process-based Project Planning. Procedia CIRP, v. 16, p. $217-$ $222,2014$.

PERUZZINI, M.; MARILUNGO, E.; GERMANI, M. Sustainable Product-Service Design in Manufacturing Industry. International Design Conference - DESIGN 2014. Anais...Dubrovnik: 2014

QU, M. et al. State-of-the-art of design, evaluation, and operation methodologies in product service systems. Computers in Industry, v. 77, n. 127, p. 1-14, 2016.

SAKAO, T.; SHIMOMURA, Y. Service Engineering: a novel engineering discipline for producers to increase value combining service and product. Journal of Cleaner Production, v. 15, n. 6, p. 590-604, 2007.

SILVA, I. S.; VELOSO, A. L.; KEATING, J. B. Focus group: Considerações teóricas e metodológicas. Revista Lusofona de Educacao, n. 26, p. 175-190, 2014.

TUKKER, A. Product services for a resource-efficient and circular economy - a review. Journal of Cleaner Production, v. 97, p. 76-91, 2015.

TUKKER, A.; TISCHNER, U. (EDS.). New Business for Old Europe: Product-service development, competitiveness and sustainability. 1. ed. Sheffield: Greenleaf Publishing, $2006 a$.

TUKKER, A.; TISCHNER, U. Product-services as a research field: past, present and future. Reflections from a decade of research. Journal of Cleaner Production, v. 14, n. 17, p. 15521556, jan. 2006b.

UNEP. Product service systemsPerspectives on Radical Changes to Sustainable .... [s.l: s.n.]. Disponível em: <http://www.risoe.dk/rispubl/art/2006_117_proceedings.pdf\#page=25>. Acesso em: 20 ago. 2014.

VAN HALEN, C. J. G.; MANZINI, E.; WIMMER, R. MEPSS - Webtool. Disponível em: <http://www.mepss.nl/>. Acesso em: 13 nov. 2015.

VASANTHA, G. V. A. et al. A review of product-service systems design methodologies. Journal of Engineering Design, v. 23, n. 9, p. 635-659, set. 2012. 